\title{
BUILD-IT: a computer vision-based interaction technique of a planning tool for construction and design
}

\author{
M. Rauterberg1, M. Bichsel², U. Leonhardt² \& M. Meier² \\ Swiss Federal Institute of Technology (ETH) \\ 1IHA \& 2IKB, Clausiusstrasse, CH-8092 Zurich, SWITZERLAND \\ http://www.ifap.bepr.ethz.ch/ rauterberg
}

\begin{abstract}
It is time to go beyond the established approaches in human-computer interaction. With the Augmented Reality (AR) design strategy humans are able to behave as much as possible in a natural way: behavior of humans in the real world with other humans and/or real world objects. Following the fundamental constraints of natural way of interacting we derive a set of recommendations for the next generation of user interfaces: the Natural User Interface (NUI). The concept of NUI is presented in form of a runnable demonstrator: a computer vision-based interaction technique for a planning tool for construction and design tasks.
\end{abstract}

KEYWORDS augmented reality, natural user interface, computer vision-based interaction

\section{INTRODUCTION}

The embodiment of computers in the work place has had a tremendous impact on the field of human-computer interaction. Mouse and graphic displays are everywhere, the desktop workstations define the frontier between the computer world and the real world. We spend a lot of time and energy to transfer information between those two worlds. This could be reduced by better integrating the virtual world of the computer with the real world of the user. Following the argumentation of Fitzmaurice, Ishii and Buxton [3] a graspable user interface has the following advantages:

"- It encourages two handed interactions; - shifts to more specialised, context sensitive input devices; • allows for more parallel input specification by the user, . leverages off of our well developed skills ... for physical object manipulations; - externalises traditionally internal computer representations; $\bullet$ facilitates interactions by making interface elements more 'direct' and more 'manipulable' by using physical artifacts; ... • affords multi-person, collaborative use" ([3] p.443).

Summarising we can conclude the following design recommendations: To empower the human to computer interaction, the user must be able to behave in a natural way bringing into action all of his or her body parts (e.g., hands, arms, face, head, voice, etc.). To interpret all of these natural expressions we need very powerful and intelligent pattern recognition techniques.

\section{THE DEMONSTRATOR "BUILD-IT"}

Inspired by the ideas of Tognazzini [5], the SUN StarFire video, and Wellner's digital desk [6] we designed a system that is based primarily on the concept of Natural User Interfaces (NUIs, see [4]). We choose the task context of planning activities for plant design. A patented system, called "BUILD-IT", has been realized and an application, supporting engineers in designing assembly lines and building plants, was implemented. The realized design room (see Figure 1) enables the users to sit around a table and to act in the interaction space with a mixture of virtual and real world objects on the table. The vertical area for (in the background of Figure 1) is used for a $3 D$ perspective view into the designed plant.

The BUILD-IT hardware has five components:

1) A table with a white surface is used as a horizontal projection and interaction area (the top view). An ASK 960 high resolution LCD projector projects the top view of the computer graphics scene vertically down to the table.

2) A white projection screen provides a vertical projection area (the side view). An ASK 860 high resolution $\mathrm{LCD}$ projector projects the $3 \mathrm{D}$ perspective view into a computer graphics scene horizontally onto the projection screen.

3) A CCD camera with a resolution of $752(\mathrm{H})$ by 582 (V) pixels looks vertically down to the table. 
4) A small brick with a special surface is the physical interaction 'device' (the universal interaction handler).

5) A low-cost Silicon Graphics Indy (IP22 $133 \mathrm{MHz}$ processor, R4600 processor, and the standard AudioVideo Board) provides the computing power for digitising the video signal coming from the camera, analysing the users' interactions on the table, and rendering the interaction result in the two views.

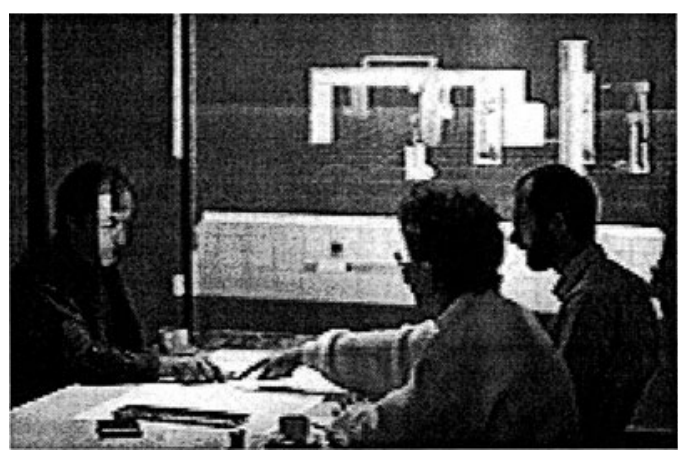

Figure 1: The design table of BUILD-IT

The BUILD-IT software consists of two independent processes communicating with each other through socket connection.

1) The real time analysis process of the video reads images from the camera, extracts contours of moving objects, analyses these contours [2], and determines the position and orientation of the universal interaction handler (the brick).

2) An application is built on top of the multi-media framework MET++ [1]. This application interprets the user action based on the position and orientation of the interaction handler, modifies a virtual scene according to the user action, and renders the top view of the new scene (for the vertical projector) and the side view into the scene (for the horizontal projector).

The application is designed for supporting providers of assembly lines and plants in the early design processes. It can read and render arbitrary CAD models of machines in VRML format. The input of a 3D model of the virtual objects is realized by connecting BUILD-IT with the CAD-System CATIA. So it was possible to import the original CAD-models into BUILD-IT.

BUILD-IT currently supports the following user (inter-)actions:

- Selection of a virtual object (e.g., a specific machine) in a 'virtual machine store' by placing the interaction handler onto the projected image of the machine in the menu area on the table.

- Positioning a machine in the virtual plant by moving the interaction handler to the corresponding position in the projected plant layout on the table. Positioning includes machine orientation that is coupled to the orientation of the interaction handler.

- Fixing the machine by covering the surface of the interaction handler with the hand and removing it.

- Re-selection of a machine by placing the interaction handler onto the specific machine.

- Deleting the machine by moving it back into menu area (the virtual machine store).

- Moving a virtual camera through the plant, rendering the perspective view of this camera, and displaying it on the vertical projection screen (the side view).

To change the side view the virtual camera can be moved on a level with the eyes of a virtual person of $1.7 \mathrm{~m}$ body height. The virtual camera is selected and moved exactly the same way as all other virtual objects. The current view position and viewing direction corresponds to the current position and orientation of the virtual camera icon in the plant layout.

The system has been empirically tested with several managers and engineers out of companies that produce assembly lines and plants. These tests showed that the system is intuitive to use, easy to learn, and that people could enjoy using it. Most persons were able to assemble virtual plants after only 30 seconds of introduction to the system.

\section{REFERENCES}

[1] Ackermann P: Developing Object-Oriented Multimedia Software Based on the MET++ Application Framework. Heidelberg: dpunkt, 1996.

[2] Bichsel M: Segmenting Simply Connected Moving Objects in a Static Scene. Transactions on Pattern Recognition and Machine Intelligence (PAMI), Vol. 16, No. 11, Nov. 1994, pp. 1138-1142.

[3] Fitzmaurice G, Ishii H, Buxton W: Bricks: Laying the Foundations for Graspable User Interfaces. In Proc. of the CHI '95, 1995, pp. 442-449.

[4] Rauterberg M, Steiger P: Pattern recognition as a key technology for the next generation of user interfaces. In Proc. of IEEE--SMC'96 (Vol. 4, 1996, pp. 2805-2810). Piscataway.

[5] Tognazzini B: Tog on Software Design. AddisonWesley, Reading MA, 1996.

[6] Wellner P: Interacting with Paper on the Digital Desk. Communications of the ACM, 36(7), 1993, pp. 87-96. 